\title{
Genetic risk scores in the prediction of plasma glucose, impaired insulin secretion, insulin resistance and incident type 2 diabetes in the METSIM study
}

\author{
Alena Stančáková ${ }^{1}$ - Teemu Kuulasmaa ${ }^{1}$. Johanna Kuusisto ${ }^{1,2}$ - Karen L. Mohlke ${ }^{3}$. \\ Francis S. Collins ${ }^{4}$ • Michael Boehnke ${ }^{5}$ - Markku Laakso ${ }^{1,2}$
}

Received: 16 December 2016 / Accepted: 28 April 2017 /Published online: 1 June 2017

(C) Springer-Verlag Berlin Heidelberg 2017

\begin{abstract}
Aims/hypothesis Many SNPs have been associated with glycaemic traits and type 2 diabetes, but their joint effects on glycaemic traits and the underlying mechanisms leading to hyperglycaemia over time are largely unknown. We aimed to investigate the association of six genetic risk scores (GRSs) with changes in plasma glucose, insulin sensitivity, insulin secretion and incident type 2 diabetes in the prospective METabolic Syndrome In Men (METSIM) study.

Methods We generated weighted GRSs for fasting plasma glucose ([FPG] $\left.\mathrm{GRS}_{\mathrm{FPG}}, 35 \mathrm{SNPs}\right), 2 \mathrm{~h}$ plasma glucose ([2hPG] GRS $\left.{ }_{2 \mathrm{hPG}}, 9 \mathrm{SNPs}\right)$, insulin secretion $\left(\mathrm{GRS}_{\mathrm{IS}}, 17\right.$ SNPs), insulin resistance $\left(\mathrm{GRS}_{\mathrm{IR}}, 9 \mathrm{SNPs}\right)$ and BMI $\left(\mathrm{GRS}_{\mathrm{BMI}}, 95 \mathrm{SNPs}\right)$ and a non-weighted GRS for type 2 diabetes $\left(\mathrm{GRS}_{\mathrm{T} 2 \mathrm{D}}, 76 \mathrm{SNPs}\right)$ in up to 8749 non-diabetic Finnish men. Linear regression was used to test associations of the GRSs with changes in glycaemic traits over time.
\end{abstract}

Electronic supplementary material The online version of this article (doi:10.1007/s00125-017-4313-4) contains peer-reviewed but unedited supplementary material, which is available to authorised users.

Markku Laakso

markku.laakso@uef.fi

1 Institute of Clinical Medicine, Internal Medicine, University of Eastern Finland, Yliopistonranta 1 B, 70210 Kuopio, Finland

2 Department of Medicine, Kuopio University Hospital, Kuopio, Finland

3 Department of Genetics, University of North Carolina, Chapel Hill, NC, USA

4 National Human Genome Research Institute, National Institutes for Health (NIH), Bethesda, MD, USA

5 Department of Biostatistics and Center for Statistical Genetics, University of Michigan, Ann Arbor, MI, USA
Results $\mathrm{GRS}_{\mathrm{T} 2 \mathrm{D}}, \mathrm{GRS}_{\mathrm{FPG}}$ and $\mathrm{GRS}_{\mathrm{IS}}$ were associated with an increase in FPG, GRS ${ }_{\mathrm{T} 2 \mathrm{D}}$ with an increase in glucose AUC and a decrease in insulin secretion, and $\mathrm{GRS}_{2 \mathrm{hPG}}$ with an increase in $2 \mathrm{hPG}$ during the follow-up ( $p<0.0017$ for all models). GRS $\mathrm{T}_{\mathrm{T} 2 \mathrm{D}}, \mathrm{GRS}_{\mathrm{FPG}}$ and $\mathrm{GRS}_{\mathrm{IS}}$ were associated with incident type 2 diabetes ( $p<0.008$ for all models). $\mathrm{GRS}_{\mathrm{BMI}}$ and $\mathrm{GRS}_{\mathrm{IR}}$ were not significantly associated with any changes in glycaemic traits.

Conclusions/interpretation In the METSIM follow-up study, $\mathrm{GRS}_{\mathrm{T} 2 \mathrm{D}}, \mathrm{GRS}_{\mathrm{FPG}}$ and $\mathrm{GRS}_{\mathrm{IS}}$ were associated with the worsening of FPG and an increase in incident type 2 diabetes. $\mathrm{GRS}_{\mathrm{T} 2 \mathrm{D}}$ was additionally associated with a decrease in insulin secretion, and $\mathrm{GRS}_{2 \mathrm{hPG}}$ with an increase in $2 \mathrm{hPG}$.

Keywords Genetic risk score · Genetic variant · Insulin resistance $\cdot$ Insulin secretion $\cdot$ Type 2 diabetes

$\begin{array}{ll}\text { Abbreviations } \\ 2 \mathrm{hPG} & 2 \mathrm{~h} \text { plasma glucose } \\ \text { FPG } & \text { Fasting plasma glucose } \\ \text { GRS } & \text { Genetic risk score } \\ \text { GRS }_{2 \mathrm{hPG}} & \text { GRS for } 2 \mathrm{hPG} \\ \text { GRS }_{\text {BMI }} & \text { GRS for BMI } \\ \text { GRS }_{\text {FPG }} & \text { GRS for FPG } \\ \text { GRS }_{\text {IR }} & \text { GRS for insulin resistance } \\ \text { GRS }_{\text {IS }} & \text { GRS for insulin secretion } \\ \text { GRS }_{\text {T2D }} & \text { GRS for type } 2 \text { diabetes } \\ \text { ISI } & \text { Index of insulin sensitivity } \\ \text { METSIM } & \text { METabolic Syndrome In Men }\end{array}$

\section{Introduction}

Genetic predisposition plays a crucial role in the risk of type 2 diabetes, in addition to environmental and lifestyle factors [1]. 
As of 2016, genome-wide association studies had identified $\sim 90$ genetic loci associated with type 2 diabetes risk and $>83$ for glycaemic traits [2]. Several of the type 2 diabetesassociated loci affect insulin secretion, and a smaller number of loci affect insulin sensitivity [3]. A total of 97 loci have been associated with BMI, an important risk factor for diabetes [4].

The effects of individual genetic variants (SNPs) on the risk of type 2 diabetes are generally small (odds ratio generally 1.15 or less), and therefore a genetic risk score (GRS) summing the effects of multiple risk alleles may provide a better tool to estimate the risk for hyperglycaemia and type 2 diabetes. Previous studies have reported that GRSs consisting of 11-65 diabetes-risk SNPs were associated with type 2 diabetes [5-14]. Only a few studies have investigated the associations of GRSs for type 2 diabetes or related traits with changes in glucose level [12, 15-17] or insulin secretion and sensitivity $[16,18]$. No prospective studies are available on the effects of the GRS for insulin secretion or insulin resistance on plasma glucose.

Determining type 2 diabetes as a dichotomous trait may underestimate the effects of genetic variants on glucose metabolism. Therefore, we investigated whether genetic variants are associated with changes in plasma glucose, insulin secretion and insulin resistance during the 4.6 year follow-up in non-diabetic individuals. To address this aim, we generated GRSs for type 2 diabetes $\left(\mathrm{GRS}_{\mathrm{T} 2 \mathrm{D}}\right.$; 76 SNPs), fasting plasma glucose ([FPG] $\mathrm{GRS}_{\mathrm{FPG}} ; 35$ SNPs), 2 h plasma glucose ([2hPG] GRS $_{2 \mathrm{hPG}}$; 9 SNPs), insulin secretion $\left(\mathrm{GRS}_{\mathrm{IS}} ; 17 \mathrm{SNPs}\right)$, insulin resistance $\left(\mathrm{GRS}_{\mathrm{IR}} ; 9\right.$ SNPs) and BMI (GRS $\mathrm{BMI} ; 95$ SNPs), and investigated their association with plasma glucose, incident type 2 diabetes and changes in insulin sensitivity and insulin secretion in the prospective METabolic Syndrome In Men (METSIM) Study.

\section{Methods}

\section{Participants}

The METSIM study comprises 10,197 Finnish men randomly selected from the population register of Kuopio town, Eastern Finland, aged from 45 to 73 years, examined in 2005-2010. The study design has been described previously [19]. The present report includes a subset of 8749 men without diabetes at the baseline visit, confirmed by a 2 h OGTT ( 75 g glucose). Glucose tolerance was evaluated according to ADA criteria [20]. The study was approved by the Ethics Committee of the University of Kuopio and Kuopio University Hospital. All study participants gave written informed consent. Two datasets from the METSIM study were used in this report, as presented below.
Data for the prospective analysis of glycaemic traits A total of 5552 non-diabetic participants at baseline have so far participated in the ongoing prospective METSIM study (mean follow-up time \pm SD: $4.6 \pm 1.0$ years) with the study protocol and measurements identical to those of the baseline study. Statistical analysis includes 5401 participants with OGTT data (17 men with missing data excluded) and without type 2 diabetes diagnosed between the baseline and follow-up visits (134 men excluded).

Data for the analysis of incident type 2 diabetes Among 8749 non-diabetic men at baseline, 693 developed incident type 2 diabetes during follow-up $(8.2 \pm 1.8$ years $)$. Diagnosis of new-onset type 2 diabetes was based on: (1) $\mathrm{FPG} \geq 7.0 \mathrm{mmol} / 1,2 \mathrm{hPG}$ in an OGTT $\geq 11.1 \mathrm{mmol} / \mathrm{l}$ or $\mathrm{HbA}_{1 \mathrm{c}} \geq 6.5 \%(47.5 \mathrm{mmol} / \mathrm{mol})$ among 5552 non-diabetic individuals who participated in the METSIM follow-up study ( $n=395)$; or (2) glucose-lowering medication started between the baseline study and 31 December 2013 ( $n=261$; information obtained from the National Drug Reimbursement registry for all 8749 participants); or (3) diagnosis by a physician based on medical records and/or $\mathrm{FPG} \geq 7.0 \mathrm{mmol} / 1$, $2 \mathrm{hPG} \geq 11.1 \mathrm{mmol} / 1$ or $\mathrm{HbA}_{1 \mathrm{c}} \geq 6.5 \%$ in outpatient/primary care laboratory measurements $(n=37)$. By aggregating data from these three sources we were able to obtain information about incident type 2 diabetes for all METSIM participants with a longer follow-up time compared with the METSIM follow-up study.

\section{Clinical and laboratory measurements}

Clinical and laboratory measurement methods have been previously published [19]. Briefly, plasma glucose was measured by enzymatic hexokinase photometric assay (Konelab Systems Reagents, Thermo Fischer Scientific, Vantaa, Finland). Insulin was determined by immunoassay (ADVIA Centaur Insulin IRI, no 02230141, Siemens Medical Solutions Diagnostics, Tarrytown, NY, USA). HDL-cholesterol and total triacylglycerol were measured by enzymatic colorimetric test (Konelab Systems Reagents). Systolic and diastolic blood pressures were calculated as an average of three measurements obtained with a mercury sphygmomanometer.

\section{Calculations}

Glucose and insulin AUCs in an OGTT were calculated by the trapezoidal method. Matsuda index of insulin sensitivity (ISI) [21] and the disposition index, a measure of insulin secretion that is the product of an OGTT-based index of early-phase insulin secretion (InsAUC ${ }_{0-30} / \mathrm{GluAUC}_{0-30}$ ) [19] and Matsuda ISI, were calculated as previously described. 


\section{Genotyping and calculation of GRSs}

A total of 216 SNPs were genotyped using either HumanOmniExpress BeadChip-12v1 (Illumina, San Diego, CA, USA; 733,202 markers) or HumanExome-12v1.1 Beadchip (Illumina, 247,870 markers). These SNPs were used to generate GRSs based on previous publications [3, 4, 9, 22]. The list of SNPs or their proxies $\left(r^{2}>0.8\right.$, used for 62 of 216 SNPs, no proxies were found for 13 SNPs) with previously published effect sizes in independent cohorts are given in electronic supplementary material [ESM] Tables 1-6. We additionally generated a GRS including 103 SNPs associated with at least one of four traits (type 2 diabetes, FPG, 2hPG and insulin secretion), and two GRSs combining SNPs associated with more than one trait (ESM Table 7). Linkage disequilibrium between the SNPs was tested using the online SNAP 2.2 tool [23]. Genotypes for all 216 SNPs were consistent with Hardy-Weinberg equilibrium at the significance level adjusted by Bonferroni method $(0.05 / 216, p>0.0002)$.

The GRSs were calculated for type 2 diabetes (76 SNPs for 8223 initially non-diabetic participants) (reviewed in Stančáková and Laakso [24]), FPG (35 SNPs, $n=8562$ ) and 2hPG (9 SNPs, $n=8610$ ) [22], insulin secretion (17 SNPs, $n=8550$ ) [9], insulin resistance (9 SNPs, $n=8592$ ) [9] and BMI (95 SNPs, $n=8133$ ) [4]. GRS FPG $_{\text {GRS }}$ hPG and $\mathrm{GRS}_{\mathrm{BMI}}$ were weighted by previously published estimated effect sizes on their respective traits [4, 22], and $\mathrm{GRS}_{\mathrm{IS}}$ and $\mathrm{GRS}_{\text {IR }}$ were weighted for their previously published estimated effect sizes on HOMA-beta and HOMA-IR indices [3, 9]. SNPs shown to be associated with other markers of insulin secretion without available effect size on HOMA-beta could not be included in the calculation of the $\mathrm{GRS}_{\text {IS }}$. For each participant, the weighted GRSs were calculated as the sum of the risk alleles of the SNPs included, where the number of the risk alleles for each SNP was multiplied by the effect size reported for that risk allele. The sum was divided by a product of the sum of all effect sizes and the number of SNPs to scale the GRSs back to the same scale as the unweighted GRSs: GRS $_{\text {weighted }}=\left[\left(\mathrm{SNP}_{1} \times\right.\right.$ effect size $\left.{ }_{1}\right)+\left(\mathrm{SNP}_{2} \times\right.$ effect size $\left._{2}\right)+\ldots+\left(\mathrm{SNP}_{\mathrm{n}} \times\right.$ effect size $\left.\left._{\mathrm{n}}\right)\right] /$ the sum of the effect sizes $\times$ the number of the SNPs. We have additionally calculated a non-weighted GRS for each trait as the sum of the risk alleles.

\section{Statistical analysis}

Statistical analyses were conducted using IBM SPSS Statistics version 21 (Chicago, IL, USA). All continuous traits except age, GRSs and change in BMI were log-transformed (inverse normal transformation gave similar results) to correct for their skewed distribution. Differences in characteristics between the groups were tested using Student's $t$ test, and differences between baseline and follow-up values by paired $t$ test. Linear regression was performed to test whether the GRSs predict changes in continuous traits during follow-up. The model was adjusted for age, BMI (except for the $\mathrm{GRS}_{\mathrm{BMI}}$ ), current smoking status (yes/no), and leisure-time physical activity (active, regular exercise at least $30 \mathrm{~min}$ a week vs inactive, occasional exercise or no exercise) at baseline, change in BMI during follow-up (to account for the change in weight during the follow-up), follow-up time (to account for the differences in the follow-up time between the participants) and the baseline level of the corresponding dependent trait. Results are presented as effect sizes in the original units (B coefficient, $\mathrm{SE}$ ) or in SD units ( $\beta$ coefficient, SE) per one risk allele. All $p$ values were calculated using log-transformed variables, but the effect sizes for FPG, 2hPG and glucose AUC were calculated using untransformed variables to obtain clinically meaningful estimates in $\mathrm{mmol} / \mathrm{l}$. Cox regression was performed to test the association of the GRSs with incident type 2 diabetes. The adjustment was made for age, BMI (except for the $\mathrm{GRS}_{\mathrm{BMI}}$ ), smoking status and physical activity at baseline, and additionally for systolic blood pressure, triacylglycerol and HDL-cholesterol to account for confounding effects of other risk factors for diabetes. Results are presented as an HR $(95 \%$ CI) per one risk allele. The level of statistical significance was Bonferroni corrected for six GRSs $(0.05 / 6$, $p<0.0083$ ) and additionally for five glycaemic traits where appropriate; $p<0.05$ was considered as nominally significant. To test whether the addition of the GRS into a prediction model consisting of clinical risk factors for type 2 diabetes (age, BMI, physical activity, smoking status, triacylglycerol, HDL-cholesterol and systolic blood pressure) improves the prediction of type 2 diabetes, we compared the AUCs of the receiver operating characteristic (ROC) curves of the models with and without the GRS.

\section{Results}

\section{Characteristics of the study participants}

Table 1 gives characteristics and the GRSs for 8749 participants who did $(n=693)$ or did not $(n=8056)$ develop type 2 diabetes during the 8.2 year follow-up, and characteristics at the baseline and 4.6 year follow-up for a subset of 5401 participants without diabetes at baseline and during follow-up who had an OGTT at both visits. At baseline, the participants who developed type 2 diabetes were significantly older, had higher BMI, higher glucose levels, lower disposition index and Matsuda ISI, were more often smokers and physically inactive, and carried a significantly higher number of the risk alleles for type 2 diabetes, FPG, $2 \mathrm{hPG}$ and insulin secretion than those who did not develop diabetes. The participants of the METSIM follow-up study had significantly higher BMI and glucose levels, and lower disposition index and Matsuda 
Table 1 Baseline clinical, laboratory characteristics and GRSs of the participants of the METSIM study who did or did not develop type 2 diabetes during the mean 8.2 year follow-up period, and changes in clinical and laboratory characteristics during the 4.6 year follow-up in participants without previously diagnosed diabetes

\begin{tabular}{|c|c|c|c|c|c|c|c|c|c|c|c|c|c|}
\hline \multirow[t]{3}{*}{ Characteristic } & \multicolumn{7}{|c|}{ Baseline characteristics of the entire cohort $(n=8749)$} & \multicolumn{6}{|c|}{ Subset with OGTT at follow-up $(n=5401)$} \\
\hline & \multicolumn{3}{|c|}{ No diabetes } & \multicolumn{3}{|c|}{ Incident type 2 diabetes } & \multirow[t]{2}{*}{$p$} & \multicolumn{3}{|c|}{ Baseline } & \multicolumn{2}{|c|}{ Follow-up } & \multirow[t]{2}{*}{$p$} \\
\hline & $n$ & Mean & SD & $n$ & Mean & SD & & $n$ & Mean & SD & Mean & SD & \\
\hline Age (years) & 8056 & 57.1 & 7.08 & 693 & 58.6 & 6.83 & $2 \times 10^{-8}$ & 5401 & 57.8 & 7.01 & 62.4 & 6.89 & \\
\hline BMI $\left(\mathrm{kg} / \mathrm{m}^{2}\right)$ & 8053 & 26.6 & 3.70 & 693 & 29.2 & 4.24 & $2 \times 10^{-50}$ & 5401 & 26.5 & 3.47 & 26.8 & 3.71 & $<0.001$ \\
\hline $\mathrm{FPG}(\mathrm{mmol} / \mathrm{l})$ & 8056 & 5.68 & 0.46 & 693 & 6.12 & 0.48 & $3 \times 10^{-112}$ & 5401 & 5.68 & 0.47 & 5.76 & 0.56 & $<0.001$ \\
\hline $2 \mathrm{hPG}(\mathrm{mmol} / \mathrm{l})$ & 8056 & 5.92 & 1.60 & 693 & 7.52 & 1.98 & $2 \times 10^{-77}$ & 5401 & 5.97 & 1.60 & 6.16 & 2.02 & $<0.001$ \\
\hline Disposition index & 8007 & 168.4 & 71.56 & 690 & 106.7 & 47.32 & $2 \times 10^{-172}$ & 5321 & 167.5 & 71.0 & 162.2 & 75.0 & $<0.001$ \\
\hline Matsuda ISI & 8007 & 7.12 & 4.16 & 690 & 4.52 & 3.17 & $5 \times 10^{-88}$ & 5321 & 7.17 & 4.08 & 6.40 & 4.31 & $<0.001$ \\
\hline Current smokers (\%) & 8056 & 17.9 & & 693 & 21.5 & & 0.020 & 5388 & 14.2 & & 11.7 & & $<0.001$ \\
\hline Physical inactivity $(\%)$ & 8056 & 34.2 & & 693 & 43.3 & & $1 \times 10^{-6}$ & 5400 & 32.5 & & 29.6 & & $<0.001$ \\
\hline $\mathrm{GRS}_{\mathrm{T} 2 \mathrm{D}}(76 \mathrm{SNPs})^{\mathrm{a}}$ & 7568 & 77.6 & 5.36 & 655 & 78.8 & 5.37 & $2 \times 10^{-7}$ & & & & & & \\
\hline $\mathrm{GRS}_{\mathrm{FPG}}(35 \mathrm{SNPs})^{\mathrm{b}}$ & 7880 & 38.0 & 4.05 & 682 & 38.5 & 3.94 & 0.001 & & & & & & \\
\hline $\mathrm{GRS}_{2 \mathrm{hPG}}(9 \mathrm{SNPs})^{\mathrm{b}}$ & 7928 & 7.47 & 1.78 & 682 & 7.62 & 1.85 & 0.044 & & & & & & \\
\hline $\mathrm{GRS}_{\mathrm{IS}}(17 \mathrm{SNPs})^{\mathrm{b}}$ & 7869 & 18.6 & 2.70 & 681 & 18.9 & 2.69 & 0.003 & & & & & & \\
\hline $\mathrm{GRS}_{\mathrm{IR}}(9 \mathrm{SNPs})^{\mathrm{b}}$ & 7909 & 9.29 & 1.93 & 683 & 9.40 & 1.92 & 0.164 & & & & & & \\
\hline $\mathrm{GRS}_{\mathrm{BMI}}(95 \mathrm{SNPs})^{\mathrm{b}}$ & 7484 & 88.1 & 6.05 & 649 & 88.5 & 6.01 & 0.095 & & & & & & \\
\hline
\end{tabular}

Glucose and insulin levels at $30 \mathrm{~min}$ of an OGTT, needed to calculate the disposition index and Matsuda ISI, were missing for 80 participants

${ }^{a}$ Non-weighted GRS

${ }^{\mathrm{b}}$ Weighted GRS

ISI at the follow-up compared with the baseline examination, although their lifestyle variables improved (reduction in the percentage of smoking and physical inactivity).

\section{Associations of GRSs with changes in glycaemic traits in the prospective analysis}

Each of the calculated GRSs was significantly associated with its corresponding trait in the cross-sectional METSIM study (all $p<0.0001$, ESM Table 8). For example, each allele of the $\mathrm{GRS}_{\mathrm{FPG}}$ and $\mathrm{GRS}_{2 \mathrm{hPG}}$ increased the levels of FPG and $2 \mathrm{hPG}$ by 0.025 and $0.087 \mathrm{mmol} / 1$, respectively.

In the prospective analysis adjusted for age, smoking status and physical activity at baseline, baseline BMI and change in BMI during follow-up (except for the GRS $\mathrm{BMI}_{\mathrm{BM}}$ ) and the follow-up time (Fig. 1, ESM Table 9), the $\mathrm{GRS}_{\mathrm{T} 2 \mathrm{D}}$ was significantly $(0.05 / 6$ GRSs $\times 5$ traits, $p<0.0017)$ associated with an increase in FPG (the effect size per a risk allele $\mathrm{B}=0.007 \mathrm{mmol} / \mathrm{l})$, glucose AUC $(\mathrm{B}=1.35 \mathrm{mmol} / \mathrm{l} \times \mathrm{min})$, and with a decrease in the disposition index (standardised effect estimate $\beta=-0.008$ ) during the 4.6 year follow-up. The $\mathrm{GRS}_{\mathrm{FPG}}$ was significantly associated with an increase in FPG $(\mathrm{B}=0.010 \mathrm{mmol} / \mathrm{l})$, and nominally $(p<0.05)$ with an increase in the glucose $\mathrm{AUC}(\mathrm{B}=0.356 \mathrm{mmol} / \mathrm{l} \times \mathrm{min})$, and a decrease in the disposition index ( $\beta=-0.007)$ during followup. The $\mathrm{GRS}_{2 \mathrm{hPG}}$ was significantly associated with an increase in $2 \mathrm{hPG}(\mathrm{B}=0.048 \mathrm{mmol} / \mathrm{l})$ during follow-up. However, the associations between the $\mathrm{GRS}_{\mathrm{FPG}}$ or $\mathrm{GRS}_{2 \mathrm{hPG}}$ and changes in their respective glucose levels were clinically minor.

$\mathrm{GRS}_{\text {IS }}$ was significantly associated with an increase in FPG ( $\mathrm{B}=0.008 \mathrm{mmol} / \mathrm{l})$ and nominally with a decrease in the disposition index $(\beta=-0.011)$ during the follow-up. $\mathrm{GRS}_{\mathrm{IR}}$ and $\mathrm{GRS}_{\mathrm{BMI}}$ were not significantly associated with changes in any of the traits. Unadjusted analyses showed similar but slightly weaker associations (ESM Table 9). We additionally performed these analyses using non-weighted GRSs and obtained very similar results (data not shown).

None of the 216 SNPs included in the GRSs was significantly associated with changes in plasma glucose, insulin secretion or insulin resistance during follow-up in 5335 participants $\left(p<4.2 \times 10^{-5}\right.$ corrected for 216 SNPs $\times 5$ traits; ESM Table 10).

\section{Associations of GRSs with incident type 2 diabetes}

$\mathrm{GRS}_{\mathrm{T} 2 \mathrm{D}}, \mathrm{GRS}_{\mathrm{FPG}}$ and $\mathrm{GRS}_{\mathrm{IS}}$ were significantly $(p<0.0083$ adjusted for six GRSs) associated with an increased risk of incident type 2 diabetes during an 8.2 year follow-up by $4 \%$, $3 \%$ and $4 \%$ per risk allele, respectively, in an unadjusted model (Table 2). The $\mathrm{GRS}_{2 \mathrm{hPG}}$ was nominally associated and GRS $_{\text {IR }}$ and GRS BMI $_{\text {BM }}$ were not significantly associated with 


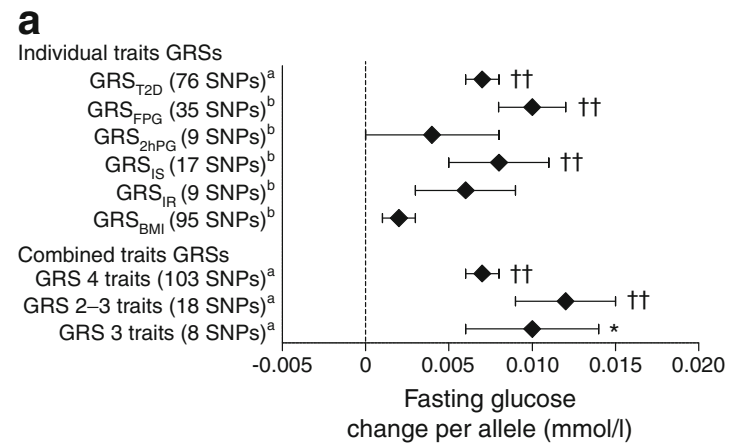

C

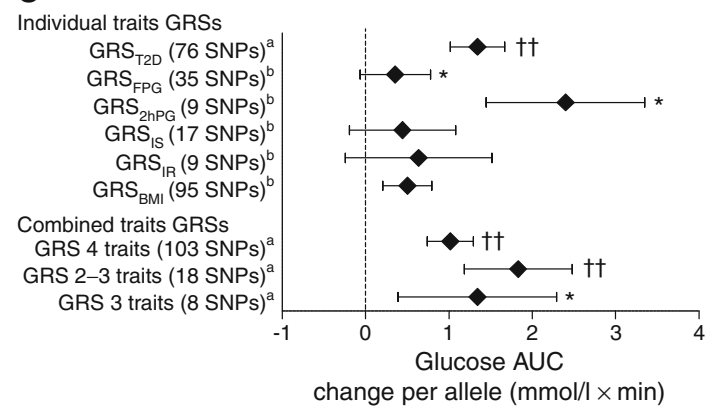

e

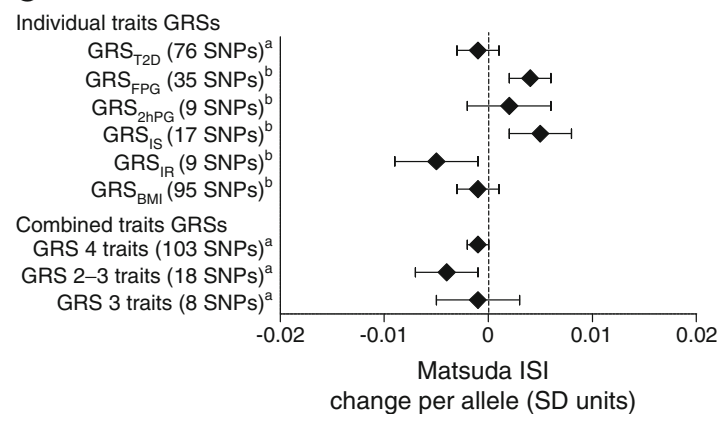

Fig. 1 Associations of $\mathrm{GRS}_{\mathrm{T} 2 \mathrm{D}}, \mathrm{GRS}_{\mathrm{FPG}}, \mathrm{GRS}_{2 \mathrm{hPG}}, \mathrm{GRS}_{\mathrm{IS}}, \mathrm{GRS}_{\mathrm{IR}}$ and $\mathrm{GRS}_{\mathrm{BMI}}$ with longitudinal changes in FPG (a), 2hPG (b), glucose AUC in an OGTT (c), insulin secretion (disposition index; d) and insulin sensitivity (Matsuda ISI; e) in the 4.6 year prospective METSIM study (maximum of 5329 men without previously diagnosed diabetes; see also

incident type 2 diabetes. After adjustment for age, BMI, smoking status and physical activity at baseline the association of $\mathrm{GRS}_{2 \mathrm{hPG}}$ became significant and other associations slightly strengthened. Further adjustment including additional risk factors for diabetes (baseline levels of systolic blood pressure, triacylglycerol and HDL-cholesterol) did not essentially affect the results. The risk of incident diabetes was $11.3 \%$ in the highest decile ( $\geq 85$ risk alleles) compared with $5.4 \%$ in the lowest decile ( $\leq 70$ risk alleles) of GRS $\mathrm{T}_{\mathrm{T} 2 \mathrm{D}}$ (Fig. 2a), and FPG increased by $0.1 \mathrm{mmol} / 1$ in the highest decile compared with $0.06 \mathrm{mmol} / \mathrm{l}$ in the lowest decile during the 4.6 year follow-up (Fig. 2b).

Based on the above results we generated an additional GRS combining SNPs associated with type 2 diabetes, FPG, $2 \mathrm{hPG}$ and insulin secretion (103 SNPs, SNPs associated with more b



d

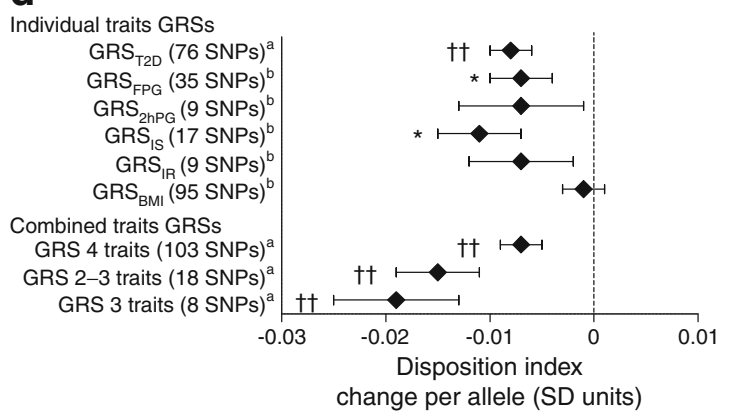

change per allele (SD units)

ESM Table 9). Results are adjusted for baseline age, smoking status, physical activity, baseline BMI and change in BMI during follow-up (except for the GRS of BMI) and the follow-up time. *Nominally significant $(p<0.05) ;{ }^{\dagger \dagger}$ significant $(p<0.0017)$. ${ }^{\mathrm{a}}$ Non-weighted GRS; $\mathrm{b}_{\text {weighted GRS }}$

than one trait or in linkage disequilibrium were only included once, preferably the SNP associated with type 2 diabetes, ESM Table 7). This 103-SNP GRS was significantly associated with incident type 2 diabetes in all models tested (Table 2).

Adding GRS $_{\text {T2D }}$ into a prediction model consisting of clinical risk factors for type 2 diabetes such as age, BMI, physical activity, smoking status, triacylglycerol, HDL-cholesterol and systolic blood pressure only slightly improved the prediction of type 2 diabetes (AUC increased from 0.711 to 0.719 , $p=0.053$ ), whereas adding the combined 103-SNP GRS to the clinical model improved the prediction of type 2 diabetes significantly (AUC 0.721, $p=0.010$ ).

We tested all 216 individual SNPs for the association with incident type 2 diabetes in up to 8619 men with available 
Table 2 Associations of GRSs with incident type 2 diabetes in an 8.2 year follow-up of the METSIM study

\begin{tabular}{|c|c|c|c|c|c|c|c|}
\hline \multirow[t]{2}{*}{ Predictor } & \multirow[t]{2}{*}{ Total/cases, $n$} & \multicolumn{2}{|l|}{ Unadjusted } & \multicolumn{2}{|l|}{ Adjusted 1} & \multicolumn{2}{|l|}{ Adjusted 2} \\
\hline & & $\mathrm{HR}(95 \% \mathrm{CI})$ & $p$ value & $\mathrm{HR}(95 \% \mathrm{CI})$ & $p$ value & $\mathrm{HR}(95 \% \mathrm{CI})$ & $p$ value \\
\hline \multicolumn{8}{|l|}{ Individual trait GRS } \\
\hline $\mathrm{GRS}_{\mathrm{T} 2 \mathrm{D}}(76 \mathrm{SNPs})^{\mathrm{a}}$ & $8223 / 655$ & $1.04(1.02,1.05)$ & $1.7 \times 10^{-7 \dagger \dagger}$ & $1.05(1.03,1.06)$ & $1.0 \times 10^{-9 \dagger \dagger}$ & $1.05(1.03,1.06)$ & $4.4 \times 10^{-10 \dagger \dagger}$ \\
\hline $\mathrm{GRS}_{\mathrm{FPG}}(35 \mathrm{SNPs})^{\mathrm{b}}$ & $8562 / 682$ & $1.03(1.01,1.05)$ & $0.001^{\dagger \dagger}$ & $1.04(1.02,1.06)$ & $6.2 \times 10^{-5 \dagger \dagger}$ & $1.04(1.02,1.06)$ & $5.4 \times 10^{-5 \dagger \dagger}$ \\
\hline $\mathrm{GRS}_{2 \mathrm{hPG}}(9 \mathrm{SNPs})^{\mathrm{b}}$ & $8610 / 682$ & $1.04(1.00,1.09)$ & $0.040^{*}$ & $1.06(1.02,1.11)$ & $0.007^{\dagger \dagger}$ & $1.06(1.01,1.10)$ & $0.009^{\dagger}$ \\
\hline $\mathrm{GRS}_{\text {IS }}(17 \mathrm{SNPs})^{\mathrm{b}}$ & $8550 / 681$ & $1.04(1.02,1.07)$ & $0.002^{\dagger \dagger}$ & $1.06(1.03,1.09)$ & $1.4 \times 10^{-4 \dagger \dagger}$ & $1.06(1.03,1.09)$ & $6.7 \times 10^{-5 \dagger \dagger}$ \\
\hline $\mathrm{GRS}_{\mathrm{IR}}(9 \mathrm{SNPs})^{\mathrm{b}}$ & $8592 / 683$ & $1.03(0.99,1.07)$ & 0.166 & $1.04(1.00,1.08)$ & 0.058 & $1.04(1.00,1.08)$ & $0.041^{*}$ \\
\hline $\mathrm{GRS}_{\mathrm{BMI}}(95 \mathrm{SNPs})^{\mathrm{b}}$ & $8133 / 649$ & $1.01(1.00,1.02)$ & 0.090 & $1.01(1.00,1.02)$ & 0.072 & $1.01(1.00,1.02)$ & 0.175 \\
\hline \multicolumn{8}{|l|}{ Combined traits GRS } \\
\hline 4 traits $(103 \mathrm{SNPs})^{\mathrm{a}}$ & $7804 / 627$ & $1.03(1.02,1.04)$ & $2.7 \times 10^{-6 \dagger \dagger}$ & $1.04(1.02,1.05)$ & $1.2 \times 10^{-8 \dagger \dagger}$ & $1.04(1.03,1.05)$ & $4.0 \times 10^{-9 \dagger \dagger}$ \\
\hline $2-3$ traits $(18 \mathrm{SNPs})^{\mathrm{a}}$ & $8582 / 682$ & $1.05(1.02,1.08)$ & $0.0016^{\dagger \dagger}$ & $1.06(1.03,1.09)$ & $1.7 \times 10^{-4 \dagger \dagger}$ & $1.06(1.03,1.09)$ & $7.1 \times 10^{-5 \dagger \dagger}$ \\
\hline 3 traits $(8 \mathrm{SNPs})^{\mathrm{a}}$ & $8605 / 684$ & $1.03(0.99,1.08)$ & 0.146 & $1.04(0.99,1.08)$ & 0.111 & $1.04(0.99,1.08)$ & 0.106 \\
\hline
\end{tabular}

The six main GRSs could be calculated for 8133-8610 participants, depending on the availability of SNPs

Data from three Cox regression models are presented: unadjusted, adjusted for age, BMI (all except for the GRS for BMI), smoking status and physical activity (adjusted model 1); and additionally adjusted for systolic blood pressure, HDL-cholesterol and triacylglycerol levels (adjusted model 2)

HRs $(95 \% \mathrm{CI})$ per risk allele are shown

*Nominally significant $(p<0.05)$

${ }^{\dagger}$ Statistically significant at the level of $p<0.0083(0.05 / 6$ main GRSs)

${ }^{a}$ Non-weighted GRS

${ }^{\mathrm{b}}$ Weighted GRS

GRS 4 traits combines the SNPs associated with at least one of the following traits: type 2 diabetes, FPG, $2 \mathrm{hPG}$ and insulin secretion; GRS 2-3 traits combines the SNPs associated with at least two of type 2 diabetes, FPG and insulin secretion and GRS 3 traits combines the SNPs associated with all three of type 2 diabetes, FPG and insulin secretion

genotypes but none of the associations was statistically significant $\left(p<2.3 \times 10^{-4}, 0.05 / 216\right)($ ESM Table 11$)$. Nominally significant associations $(p<0.05)$ were observed for type 2 diabetes-associated SNPs in TCF7L2 and THADA, and several BMI-associated SNPs, where the BMI-increasing allele was associated with either higher (SNPs in/near ETV5, ETS2, $G R P, P R K D 1, E H B P 1)$ or lower (SNPs in/near $K C N K 3$, $K C T D 15, F U B P 1, M C 4 R, L O C 100287559)$ risk of incident diabetes.

\section{GRSs including shared SNPs}

Several GRSs showing significant associations with incident type 2 diabetes and changes in glycaemic traits during the follow-up share several SNPs (or different SNPs from the same genetic loci, mostly representing proxies) (ESM Table 12). We additionally generated two GRSs combining SNPs associated with two (18 SNPs) or all three (8 SNPs) of the following traits: type 2 diabetes, FPG and insulin secretion (ESM Table 7). The 18-SNP GRS was significantly associated with incident type 2 diabetes, and both 18-SNP and 8-SNP GRSs were significantly or nominally associated with changes in FPG, glucose AUC and insulin secretion over time. This suggests that the overlapping SNPs contribute to the associations observed for the $\mathrm{GRS}_{\mathrm{T} 2 \mathrm{D}}, \mathrm{GRS}_{\mathrm{FPG}}$ and $\mathrm{GRS}_{\mathrm{IS}}$ (Table 2 and ESM Table 9).

\section{Discussion}

The main findings of our prospective study are that: (1) $\mathrm{GRS}_{\mathrm{T} 2 \mathrm{D}}, \mathrm{GRS}_{\mathrm{FPG}}$ and $\mathrm{GRS}_{\mathrm{IS}}$ were significantly associated with an increase in FPG during the follow-up, and $\mathrm{GRS}_{\mathrm{T} 2 \mathrm{D}}$ was additionally significantly associated with changes in the glucose AUC and insulin secretion (disposition index); (2) $\mathrm{GRS}_{2 \mathrm{hPG}}$ was associated with an increase in $2 \mathrm{hPG}$ level; (3) $\mathrm{GRS}_{\mathrm{T} 2 \mathrm{D}}, \mathrm{GRS}_{\mathrm{FPG}}$ and GRS $\mathrm{GS}_{\mathrm{IS}}$ were significantly associated with the incident type 2 diabetes; and (4) $\mathrm{GRS}_{\mathrm{BMI}}$ and $\mathrm{GRS}_{\mathrm{IR}}$ were not significantly associated with changes in glycaemic traits.

\section{GRS $_{\text {FPG }}$ and GRS $_{2 \mathrm{hPG}}$}

Identifying individuals at high risk of developing type 2 diabetes is critical for effective prevention of this disease. Glucose levels are the strongest predictors for type 2 diabetes [1, 25], but none of the previous studies has investigated the association of genetic variants with glucose AUC in an OGTT (based on 0, 30 and 120 min glucose 

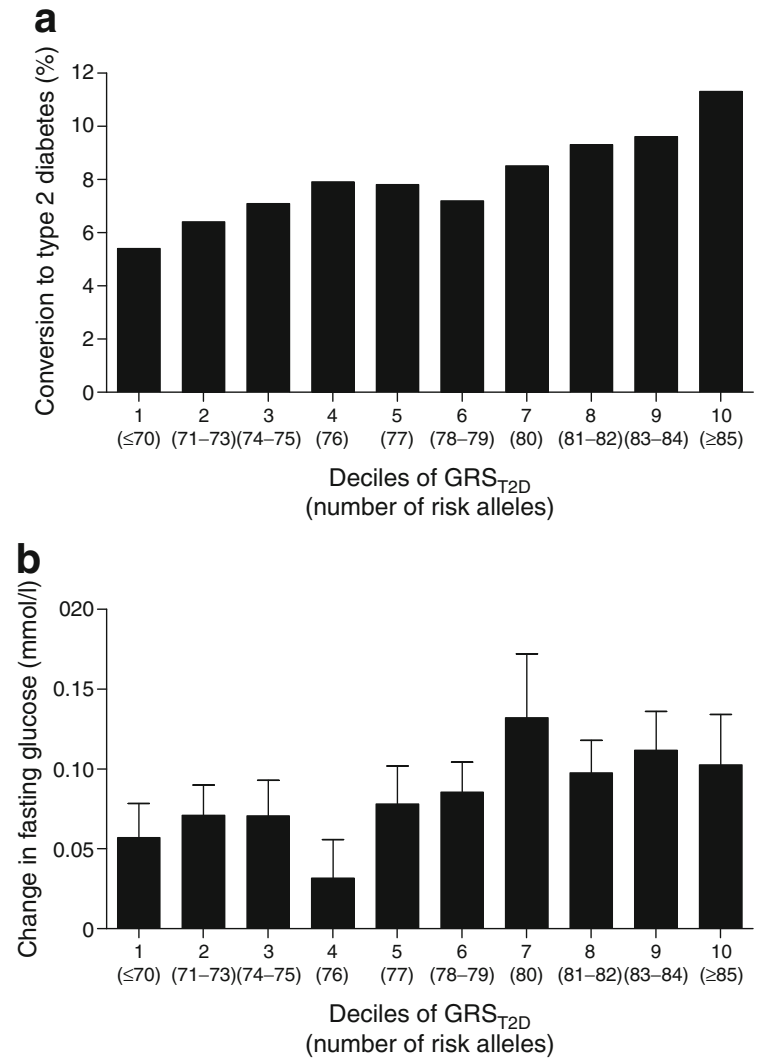

Fig. 2 (a) Conversion to type 2 diabetes (\%) during an 8.2 year followup in 8223 METSIM participants without diabetes at entry according to GRS $_{\text {T2D }}$ decile. Number of individuals in each decile group: (1) 726 ; (2) 1040; (3) 955; (4) 597; (5) 603; (6) 1269; (7) 567; (8) 936; (9) 716; and (10) 814. (b) Change in fasting glucose during a 4.6 year follow-up in 5076 participants without previously diagnosed diabetes according to GRS $_{\text {T2D }}$ decile. Number of individuals in each decile group: (1) 471 ; (2) 630 ; (3) 592 ; (4) 381 ; (5) 386; (6) 763; (7) 343; (8) 590; (9) 423; and $(10) 497$

levels), which reflects the total 'glucose burden' on pancreatic beta cells. A Swedish study showed that a 16-SNP GRS for FPG was associated with higher FPG levels $(0.030 \mathrm{mmol} / 1$ per GRS unit) and $2 \mathrm{hPG}$ levels $(0.026 \mathrm{mmol} / \mathrm{l}$ per GRS unit) at baseline, and an elevation of FPG levels from baseline to follow-up [26]. Another study reported an association of a 16-SNP GRS for FPG with higher FPG levels $(0.029 \mathrm{mmol} / \mathrm{l}$ per GRS unit) that remained consistent over time, and an association of a 5SNP GRS for $2 \mathrm{hPG}$ with higher $2 \mathrm{hPG}$ levels $(0.076 \mathrm{mmol} / \mathrm{l}$ per GRS unit) which became stronger with increasing age [15]. Our study demonstrated that the $\mathrm{GRS}_{\mathrm{FPG}}(35 \mathrm{SNPs})$ and the $\mathrm{GRS}_{2 \mathrm{hPG}}(9 \mathrm{SNPs})$ had similar effect sizes on FPG $(0.025 \mathrm{mmol} / 1$ per GRS unit $)$ and $2 \mathrm{hPG}(0.087 \mathrm{mmol} / 1$ per GRS unit) levels at baseline, respectively, as in earlier studies $[15,26]$. Moreover, the $\mathrm{GRS}_{\mathrm{FPG}}$ and the $\mathrm{GRS}_{2 \mathrm{hPG}}$ were significantly associated with further increases in FPG (by $0.010 \mathrm{mmol} / 1$ per GRS unit) and $2 \mathrm{hPG}$ (by $0.048 \mathrm{mmol} / \mathrm{l}$ per GRS unit), respectively, and nominally with an increase in glucose AUC during the follow-up, even after correcting for baseline age, BMI, smoking status, physical activity, corresponding baseline glucose level, change in BMI during the follow-up, and follow-up time. Finally, both GRS $_{\mathrm{FPG}}$ and $\mathrm{GRS}_{2 \mathrm{hPG}}$ were associated with an increased risk of incident type 2 diabetes, in contrast to a previous study using a 24-SNP GRS for FPG that did not show significant association [12]. These findings suggest that genetic factors predict increases in glucose levels and glucose AUC, explaining, at least in part, the conversion to type 2 diabetes. However, these effects may be too modest to be clinically relevant.

\section{GRS $_{\text {IS }}$ and GRS $_{\text {IR }}$}

In our study, $\mathrm{GRS}_{\mathrm{IS}}$ was significantly associated with an increase in FPG, a decrease in disposition index (nominally significant) and an increased risk of incident type 2 diabetes during follow-up. These findings emphasise the central role of impaired insulin secretion in the conversion to diabetes. By contrast, $\mathrm{GRS}_{\mathrm{IR}}$ did not significantly predict changes in FPG or $2 \mathrm{hPG}$, or the conversion to diabetes. This is consistent with a previous study [9] and suggests that insulin resistance is mostly acquired (weight gain, lack of exercise, unhealthy diet), and not genetically determined to the extent of insulin secretion [25]. However, a previous large study $(18,565$ individuals including 8124 incident cases of diabetes) demonstrated that the GRS for insulin resistance was associated with incident type 2 diabetes [27], which may indicate that our study was underpowered to demonstrate this association.

\section{GRS $_{\text {T2D }}$}

Our GRS $\mathrm{T}_{\mathrm{T} 2 \mathrm{D}}$ including 76 SNPs was significantly associated with an increased risk of incident type 2 diabetes that was higher by twofold in the highest decile compared with the lowest decile. Accordingly, FPG was increased by $0.1 \mathrm{mmol} / 1$ in the highest decile compared with a $0.06 \mathrm{mmol} / 1$ increase in the lowest decile during the 4.6 year follow-up. The increase in the risk of diabetes was $5 \%$ per GRS $_{\text {T2D }}$ unit in the adjusted model, which is slightly less than the $8 \%$ reported in a previous study using a 62 -SNP GRS for type 2 diabetes [9], and in studies including a lower number of type 2 diabetes-associated SNPs [5-13, 16, 28].

Only a few previous studies have investigated the associations of diabetes-associated SNPs with longitudinal changes in glycaemic traits [15-16, 26, 29]. A Danish study using a GRS based on 46 diabetes-associated SNPs reported a significant increase in glucose levels in an OGTT and a decrease in OGTT-based insulin secretion indices and the disposition index per risk allele during a 5 year follow-up [16]. In a 9 year follow-up of a Chinese study, a GRS based on 40 diabetes- 
associated SNPs predicted deterioration in beta cell function but not insulin sensitivity (assessed by the OGTT-based indices) [18]. Our results of the associations of $\mathrm{GRS}_{\mathrm{T} 2 \mathrm{D}}$ with incident diabetes and with changes in glucose levels (FPG, glucose AUC) and insulin secretion during the follow-up are consistent with these results.

\section{GRS $_{\text {BMI }}$}

Obesity is an important risk factor for diabetes. Our study did not show significant association of $\mathrm{GRS}_{\mathrm{BMI}}(95 \mathrm{SNPs})$ with incident type 2 diabetes and changes in plasma glucose, insulin secretion (disposition index) or insulin sensitivity (Matsuda ISI) during the follow-up. In the GLACIER study, no significant association of a 97-SNP GRS for BMI was observed with FPG or $2 \mathrm{hPG}$ during a 10 year follow-up [29]. By contrast, a cross-sectional Chinese study reported that a GRS consisting of 30 BMI-associated SNPs significantly increased the risk of type 2 diabetes [30].

The strength of our study is that it investigates several GRSs relevant for glucose metabolism for associations with trait changes over time in a single population. It shows that although the GRSs specific for FPG and 2hPG are the best predictors of the changes in the respective glucose levels, the GRS for type 2 diabetes, including the highest number of risk variants published so far, significantly predicts changes in several traits including fasting glucose, glucose AUC, insulin secretion and incident type 2 diabetes. Nevertheless, it contributed relatively little to a diabetes-prediction model that includes clinical risk factors, similar to previous studies including a smaller number of SNPs $[5,9,11,28]$, suggesting that the clinical relevance of the current GRSs remains limited. However, the SNPs used in the current analyses were originally identified in cross-sectional studies, and longitudinal studies may provide more knowledge of the genetics of trait changes over time. Furthermore, including a larger number of SNPs in the GRSs will likely improve their predictive ability, as supported by our observation that the combined 103-SNP GRS significantly improved the prediction of type 2 diabetes. Other strengths of our study are a large and homogeneous population, detailed phenotyping including measures of insulin secretion and insulin resistance at both baseline and followup examinations, and extensive genotyping, which allowed us to use the most complete up-to-date genetic associations with type 2 diabetes and related traits. A limitation of our study is that, as we included only Finnish men, we cannot ensure the applicability of our results to women or other populations.

In conclusion, our study demonstrates that $\mathrm{GRS}_{\mathrm{T} 2 \mathrm{D}}$, $\mathrm{GRS}_{\mathrm{FPG}}$ and GRS $\mathrm{SIS}_{\text {s }}$ predicted the worsening of FPG and incident type 2 diabetes, with $\mathrm{GRS}_{\mathrm{T} 2 \mathrm{D}}$ also predicting adverse changes in insulin secretion. These GRSs provide a useful tool to estimate the effects of multiple risk alleles on the development of hyperglycaemia and type 2 diabetes.
Data availability The datasets generated during and/or analysed during the current study are not publicly available for reasons related to privacy and participant consent but are available from the corresponding author on reasonable request.

Funding This work has been supported by the grants from the Academy of Finland (to ML and AS), the Finnish Diabetes Research Foundation (ML), the Finnish Cardiovascular Research Foundation (ML), Strategic Research Funding from the University of Eastern Finland, Kuopio (ML), EVO Grant 5263 from the Kuopio University Hospital (ML), the Sigrid Juselius Foundation (ML), support from the $\mathrm{NIH}$ intramural research programme (FSC) and NIH grants DK062370 (MB), DK072193 (KLM) and DK093757 (KLM). The funding bodies named had no role in the design and conduct of the study, collection management, analysis and interpretation of the data, preparation review or approval of the manuscript.

Duality of interest The authors declare that there is no duality of interest associated with this manuscript.

Contribution statement AS and ML wrote the manuscript and researched the data. AS performed the statistical analyses. ML, JK, AS and TK designed the study and reviewed and edited the manuscript. MB, FSC and KLM contributed to genotyping and reviewed and edited the manuscript. All authors approved the final version of the manuscript. ML is the guarantor of this work and, as such, had full access to all the data in the study and takes responsibility for the integrity of the data and the accuracy of the data analysis.

\section{References}

1. Alberti G, Zimmet P, Shaw J et al (2004) Type 2 diabetes in the young: the evolving epidemic: the international diabetes federation consensus workshop. Diabetes Care 27:1798-1811

2. Mohlke KL, Boehnke M (2015) Recent advances in understanding the genetic architecture of type 2 diabetes. Hum Mol Genet 24: R85-R92

3. Dimas AS, Lagou V, Barker A et al (2014) Impact of type 2 diabetes susceptibility variants on quantitative glycemic traits reveals mechanistic heterogeneity. Diabetes 63:2158-2171

4. Locke AE, Kahali B, Berndt SI et al (2015) Genetic studies of body mass index yield new insights for obesity biology. Nature 518:197-206

5. Lyssenko V, Jonsson A, Almgren $P$ et al (2008) Clinical risk factors, DNA variants, and the development of type 2 diabetes. N Engl J Med 359:2220-2232

6. Meigs JB, Shrader P, Sullivan LM et al (2008) Genotype score in addition to common risk factors for prediction of type 2 diabetes. $\mathrm{N}$ Engl J Med 359:2208-2219

7. van Hoek M, Dehghan A, Witteman JC et al (2008) Predicting type 2 diabetes based on polymorphisms from genome-wide association studies: a population-based study. Diabetes 57:3122-3128

8. Vassy JL, Dasmahapatra P, Meigs JB et al (2012) Genotype prediction of adult type 2 diabetes from adolescence in a multiracial population. Pediatrics 130:e1235-e1242

9. Vassy JL, Hivert MF, Porneala B et al (2014) Polygenic type 2 diabetes prediction at the limit of common variant detection. Diabetes 63:2172-2182

10. Talmud PJ, Hingorani AD, Cooper JA et al (2010) Utility of genetic and non-genetic risk factors in prediction of type 2 diabetes: Whitehall II prospective cohort study. BMJ 340:b4838

11. Talmud PJ, Cooper JA, Morris RW et al (2015) Sixty-five common genetic variants and prediction of type 2 diabetes. Diabetes 64: $1830-1840$ 
12. Vaxillaire M, Yengo L, Lobbens $\mathrm{S}$ et al (2014) Type 2 diabetesrelated genetic risk scores associated with variations in fasting plasma glucose and development of impaired glucose homeostasis in the prospective DESIR study. Diabetologia 57:1601-1610

13. Walford GA, Porneala BC, Dauriz M et al (2014) Metabolite traits and genetic risk provide complementary information for the prediction of future type 2 diabetes. Diabetes Care 37:2508-2514

14. Poveda A, Koivula RW, Ahmad S et al (2016) Innate biology versus lifestyle behaviour in the aetiology of obesity and type 2 diabetes: the GLACIER study. Diabetologia 59:462-471

15. Jensen AC, Barker A, Kumari M et al (2011) Associations of common genetic variants with age-related changes in fasting and postload glucose: evidence from 18 years of follow-up of the Whitehall II cohort. Diabetes 60:1617-1623

16. Andersson EA, Allin KH, Sandholt CH et al (2013) Genetic risk score of 46 type 2 diabetes risk variants associates with changes in plasma glucose and estimates of pancreatic $\beta$-cell function over 5 years of follow-up. Diabetes 62:3610-3617

17. Go MJ, Lee Y, Park S, Kwak SH, Kim BJ, Lee J (2016) Geneticrisk assessment of GWAS-derived susceptibility loci for type 2 diabetes in a 10 year follow-up of a population-based cohort study. J Hum Genet 61:1009-1012

18. Yan J, Peng D, Jiang F et al (2016) Impaired pancreatic beta cell compensatory function is the main cause of type 2 diabetes in individuals with high genetic risk: a 9 year prospective cohort study in the Chinese population. Diabetologia 59:1458-1462

19. Stančáková A, Javorský M, Kuulasmaa T, Haffner SM, Kuusisto J, Laakso M (2009) Changes in insulin sensitivity and insulin release in relation to glycemia and glucose tolerance in 6,414 Finnish men. Diabetes 58:1212-1221

20. Genuth S, Alberti KG, Bennett P et al (2003) Follow-up report on the diagnosis of diabetes mellitus. Diabetes Care 26:3160-3167
21. Matsuda M, DeFronzo RA (1999) Insulin sensitivity indices obtained from oral glucose tolerance testing: comparison with the euglycemic insulin clamp. Diabetes Care 22:1462-1470

22. Scott RA, Lagou V, Welch RP et al (2012) Large-scale association analyses identify new loci influencing glycemic traits and provide insight into the underlying biological pathways. Nat Genet 44:991-1005

23. Johnson AD, Handsaker RE, Pulit S, Nizzari MM, O'Donnell CJ, de Bakker PIW (2008) SNAP: a web-based tool for identification and annotation of proxy SNPs using HapMap. Bioinformatics 24: 2938-2939

24. Stančáková A, Laakso M (2016) Genetics of type 2 diabetes. Endocr Dev 31:203-220

25. Katoh S, Lehtovirta M, Kaprio J et al (2005) Genetic and environmental effects on fasting and postchallenge plasma glucose and serum insulin values in Finnish twins. J Clin Endocrinol Metab 90:2642-2647

26. Renström F, Shungin D, Johansson I et al (2011) Genetic predisposition to long-term nondiabetic deteriorations in glucose homeostasis: tenyear follow-up of the GLACIER study. Diabetes 60:345-354

27. Scott RA, Fall T, Pasko D et al (2014) Common genetic variants highlight the role of insulin resistance and body fat distribution in type 2 diabetes, independent of obesity. Diabetes 63:4378-4387

28. de Miguel-Yanes JM, Shrader P, Pencina MJ et al (2011) Genetic risk reclassification for type 2 diabetes by age below or above 50 years using 40 type 2 diabetes risk single nucleotide polymorphisms. Diabetes Care 34:121-125

29. Ahmad S, Poveda A, Shungin D et al (2016) Established BMIassociated genetic variants and their prospective associations with BMI and other cardiometabolic traits: the GLACIER study. Int J Obes 40:1346-1352

30. Zhu J, Zong G, Lu L et al (2014) Association of genetic predisposition to obesity with type 2 diabetes risk in Han Chinese individuals. Diabetologia 57:1830-1833 